\section{Stellungnahme der DGPPN}

Derzeit werden die Diagnosemanuale für psychische Erkrankungen überarbeitet. Zum einen wird in Kürze das US-amerikanische Diagnosemanual DSM-5 erwartet, in zwei Jahren folgt die internationale Klassifikation ICD-11. Dabei werden Konzepte, Grenzen und Definitionen psychischer Erkrankungen einer Revision unterzogen. In einer Stellungnahme zum DSM-5 warnt die Deutsche Gesellschaft für Psychiatrie, Psychotherapie und Nervenheilkunde (DGPPN) davor, die Änderungen der US-Kollegen kritiklos zu übernehmen. Diagnosen sollten sich auch weiterhin nur auf medizinisch relevante Leiden beziehen.

Die ausführliche Stellungnahme lesen Sie unter: www.dgppn.de/fileadmin/ user_upload/_medien/download/pdf/stellungnahmen/2013/DGPPNStellungnahme_DSM-5_Final.pdf

\section{Kritik auch von US-Psychiatern}

Kritik am DSM-5 wird auch von US-Psychiatern laut, unter anderem vom inzwischen emeritierten Professor Allen Frances. Der Psychiater hat selbst an der dritten Ausgabe des Diagnosehandbuchs mitgewirkt und die Revision zur vierten Ausgabe geleitet. Seit einigen Jahren fällt er jedoch mit negativen Äußerungen zur anstehenden Neuausgabe auf. Zuletzt tourte Frances auch durch Deutschland und gab zahlreiche Interviews zum DSM-5 und zu seinem neuen Buch. Dabei nahm er etwa die Ausweitung der Diagnosen bei Kindern aufs Korn. So könnten heftige Wutausbrüche seiner Auffassung nach künftig unter "affektive Dysregulation“ (disruptive mood dysregulation disorder) fallen. Auch wenn es dafür keine zugelassenen Therapien gibt, sieht Frances die Gefahr, "dass auch diese Kinder mit Antipsychotika behandelt werden". Ebenfalls kritisch sieht Frances die Praxis in den USA, nach der inzwischen Haus- ärzte, Kinderärzte und Internisten 80 \% der Psychopharmaka verordnen. „Diese Verschreibungen erfolgen in der Regel nach einem einzigen Besuch an einem Tag, an dem es den Patienten besonders schlecht geht", so der Psychiater im Interview mit der "Frankfurter Allgemeinen Sonntagszeitung". Schließlich beklagt er die dünne wissenschaftliche Basis der bisherigen Therapien: Wir seien immer noch weit von einem Verständnis dessen entfernt, was bei psychischen Krankheiten im Gehirn passiert.

In diese Kerbe schlägt auch der Direktor des National Institute of Mental Health (NIMH), Dr. Thomas Insel: „Das DSM ist weiterhin das beste Werkzeug für Kliniker, die Patienten behandeln, aber es bildet die Komplexität vieler Krankheiten nicht ab", so Insel in der "New York Times". Die Art und Weise, wie psychische Krankheiten im Manual kategorisiert werden, dürfe die Forschung nicht beeinflussen. "Solange die Wissenschaftsgemeinde das DSM als Bibel betrachtet, machen wir keinen Fortschritt." Insel sorgte für Aufregung, als er vor kurzem ankündigte, das NIMH werde sich bei seinen Projekten von den DSM-Diagnosen wegbewegen und eigene Forschungskriterien aufstellen, weil "die Patienten Besseres verdienten".

Dr. Jeffrey Lieberman von der American Psychiatric Association verteidigte dagegen das neue Manual. Es basiere auf der Forschung der vergangenen 20 Jahre und werde letztlich die Versorgung der Patienten verbessern. "Natürlich wäre uns nichts lieber als noch bessere wissenschaftliche Erkenntnisse“, wird er in der "New York Times" zitiert.

"Nicht jeder Wutanfall ist eine Psychose". Interview mit Professor Allen Frances in der "Frankfurter Allgemeinen Sonntagszeitung", 21.4.2013

Psychiatry's Guide Is Out of Touch With Science, Experts Say. New York Times, 6. Mai 2013 mit wird ein Anreiz in Richtung Psychotherapie nach Richtlinienverfahren gesetzt. Das ist in den USA bei anderen Honorarregelungen nicht regelhaft so.

InFoNP: Ist das nicht eher ein Problem der Versorgungsstrukturen und weniger der ärztlichen Diagnosen?

Maier: Ärztliche Diagnosen sollen den ärztlichen Hilfebedarf kennzeichnen. Wenn man die Grenze für psychische Krankheiten noch weiter zieht, und etwa alle Trauernden zu Kranken mit Leistungsansprüchen macht, dann brechen die sozialen Sicherungssysteme aufgrund des umfänglich gewachsenen, geforderten Hilfebedarfs zusammen.

InFoNP: Die DGPPN kritisiert auch, dass im DSM-5 altersbedingte Leistungseinschränkungen Krankheitswert bekommen, etwa die "leichte neurokognitive Störung". Könnte eine solche Diagnose nicht helfen, eine klinisch manifeste Demenz besser abzugrenzen? Maier: Sie stellen also fest, jemand hat im Alter eine unterdurchschnittliche Gedächtnisleistung. Und nun? Man sagt ihm, er ist krank und muss zum Arzt gehen, der kann aber gar nichts machen. Vielleicht wird das mal eine Demenz. Aber dann muss man ihn doch nicht jetzt schon für krank erklären, insbesondere dann nicht, wenn man diesen ungünstigen Verlauf zur Demenz nicht verhindern kann. Ich kann ihm sagen, wahrscheinlich bleibt es eine Altersvergesslichkeit, zu $40 \%$ wird es in vier Jahren eine Demenz. Erhöhtes Risiko dürfen Ärzte doch nicht schon als Krankheit definieren. Durch Zurechnung von Risikostadien zu Krankheiten lässt sich vielleicht die Bedeutsamkeit eines Fachs hervorheben oder die Wichtigkeit einzelner Proponenten für diesen Schritt unterstreichen. Betroffene haben dadurch jedenfalls sicher keinen Nutzen, wenn es keine präventiven Therapien gibt. Solange es die nicht gibt, muss man zurückhaltend sein, sonst ist der Schaden größer als der Nutzen.

InFoNP: Der DSM-5 macht das ja auch mit Blick auf künftige Therapien. Es heißt dann zum Beispiel "leichte kognitive Störung assoziiert mit einer Alzheimer-Erkrankung", sofern außer den klinischen Befunden auch noch Biomarker für eine Alzheimer-Pathologie sprechen.

Maier: Wenn sie eine Alzheimer-Pathologie bei leichter kognitiver Störung sichern können, ist das eine andere Sache. Sie erhöhen damit erheblich die Spezifität. Wir sind aber weit davon entfernt, dass jeder mit Altersvergesslichkeit ein Amyloid-PET oder eine Liquorpunktion erhält. Solange es keine wirksamen Therapien gibt, wäre das auch gar nicht sinnvoll.

InFoNP: Befürchten Sie, dass wesentliche Änderungen im DSM-5 später auch im ICD-11 stehen?

Maier: Die Kritik am DSM-5 in den USA ist inzwischen so massiv, dass ich mir nicht vorstellen kann, dass das einfach so übernommen wird.

\section{Herzlichen Dank für das Gespräch!}

\section{Das Interview führte Thomas Müller.}

